\title{
Acoustic Coherent Backscatter Enhancement from Aggregations of Point Scatterers
}

\author{
David R. Dowling \\ Department of Mechanical Engineering \\ University of Michigan \\ Ann Arbor, MI 48109-2133 \\ phone: (734) 936-0423 fax: (734) 764-4256 email: drd@umich.edu \\ Award \#: N00014-11-1-0258 \\ http://www.personal.engin.umich.edu/ drd/
}

\section{LONG-TERM GOALS}

The overall long-term goal for this project is to determine if and how acoustic coherent backscatter enhancement (ACBE) can be used for classification of active sonar returns in a wide variety of ocean environments. During its first three years, this project has focused on simulations of acoustic multiple scattering from two- and now three-dimensional aggregations of omni-directional point scatterers to determine the parametric realms in which ACBE might be observed, and its characteristics when it is observed.

\section{OBJECTIVES}

The detailed objectives of the current research effort are to determine the parametric dependence of ACBE peak amplitude, peak emergence rate as the number of observations increases, peak angular width, and peak time dependence. Here the independent parameters are the range between the scattering aggregation and the receiving array, the receiving array characteristics, incident wave characteristics (wave front shape, waveform, frequency, bandwidth, duration), and aggregation characteristics (scatterer cross section and mean spacing, overall aggregation size and shape, etc.). Eventually, underwater waveguide characteristics will be considered as well.

\section{APPROACH}

The current approach involves numerical evaluation of the fundamental equations of multiple scattering from an aggregation of omni-directional point scatterers ${ }^{1}$. If $\psi(\mathbf{r})$ is the harmonic acoustic pressure field at frequency $\omega$ at the point $\mathbf{r}$ and $\psi_{0}(\mathbf{r})$ is the harmonic field incident on the aggregation of scatterers located at $\mathbf{r}_{n}$, then

$$
\psi(\mathbf{r})=\psi_{0}(\mathbf{r})+\psi_{s}(\mathbf{r})=\psi_{0}(\mathbf{r})+\sum_{n=1}^{N} g_{n} \psi_{n}\left(\mathbf{r}_{n}\right) G\left(\mathbf{r}, \mathbf{r}_{n}\right),
$$

where $\psi_{C}(\mathbf{r})$ is the scattered field and is given by the sum in (1), $N$ is the number of scatterers, $g_{n}$ is the scattering coefficient of the $n^{\text {th }}$ scatterer, $\psi_{n}\left(\mathbf{r}_{n}\right)$ is the field incident on the $n^{\text {th }}$ scatterer,

$$
\psi_{n}\left(\mathbf{r}_{n}\right)=\psi_{0}\left(\mathbf{r}_{n}\right)+\sum_{j=1, j \neq n}^{N} g_{j} \psi_{j}\left(\mathbf{r}_{j}\right) G\left(\mathbf{r}_{n}, \mathbf{r}_{j}\right)
$$




\section{Report Documentation Page}

Form Approved

OMB No. 0704-0188

Public reporting burden for the collection of information is estimated to average 1 hour per response, including the time for reviewing instructions, searching existing data sources, gathering and maintaining the data needed, and completing and reviewing the collection of information. Send comments regarding this burden estimate or any other aspect of this collection of information,

including suggestions for reducing this burden, to Washington Headquarters Services, Directorate for Information Operations and Reports, 1215 Jefferson Davis Highway, Suite 1204, Arlington

VA 22202-4302. Respondents should be aware that notwithstanding any other provision of law, no person shall be subject to a penalty for failing to comply with a collection of information if it

does not display a currently valid OMB control number.

1. REPORT DATE

30 SEP 2014

4. TITLE AND SUBTITLE

Acoustic Coherent Backscatter Enhancement from Aggregations of Point Scatterers

6. AUTHOR(S)

7. PERFORMING ORGANIZATION NAME(S) AND ADDRESS(ES)

University of Michigan,Department of Mechanical Engineering,Ann Arbor,MI,48109

9. SPONSORING/MONITORING AGENCY NAME(S) AND ADDRESS(ES)

3. DATES COVERED

00-00-2014 to 00-00-2014

5a. CONTRACT NUMBER

5b. GRANT NUMBER

5c. PROGRAM ELEMENT NUMBER

5d. PROJECT NUMBER

5e. TASK NUMBER

5f. WORK UNIT NUMBER

8. PERFORMING ORGANIZATION

REPORT NUMBER

10. SPONSOR/MONITOR'S ACRONYM(S)

11. SPONSOR/MONITOR'S REPORT

NUMBER(S)

12. DISTRIBUTION/AVAILABILITY STATEMENT

Approved for public release; distribution unlimited

13. SUPPLEMENTARY NOTES

14. ABSTRACT

15. SUBJECT TERMS

16. SECURITY CLASSIFICATION OF:

a. REPORT

unclassified b. ABSTRACT

unclassified c. THIS PAGE

unclassified
17. LIMITATION OF ABSTRACT

Same as

Report (SAR)
18. NUMBER 19a. NAME OF

OF PAGES RESPONSIBLE PERSON

6

Standard Form 298 (Rev. 8-98) Prescribed by ANSI Std Z39-1 
and $G\left(\mathbf{r}_{n}, \mathbf{r}_{j}\right)$ is the free-space Green's function between the locations $\mathbf{r}_{n}$ and $\mathbf{r}_{j}$,

$$
G\left(\mathbf{r}_{n}, \mathbf{r}_{j}\right)=\frac{\exp \left\{-i k_{0} \mid \mathbf{r}_{n}-\mathbf{r}_{j}\right\}}{\left|\mathbf{r}_{n}-\mathbf{r}_{j}\right|}
$$

where $k_{0}=\omega / c$ is the wave number magnitude of the incident field, $c$ is the sound speed, and $i=\sqrt{-1}$. When the incident field and the scattering coefficients are known, (2) can be written $N$ times, once for each scatterer $1 \leq n \leq N$, and these $N$ algebraic equations can be solved to determine $\psi_{n}\left(\mathbf{r}_{n}\right)$. The total field at any location is then recovered from (1) and (3) using the known $\psi_{0}(\mathbf{r})$, the known $g_{n}$, and the calculated $\psi_{n}\left(\mathbf{r}_{n}\right)$. This formulation is akin to the direct boundary-integral formulation of computational acoustics with one computational element assigned to each scatterer. The computational burden of this approach is set by the inversion of the fully-populated $N$-by- $N$ algebraic system that determines $\psi_{n}\left(\mathbf{r}_{n}\right)$.

In the current investigation, the scatterers are placed randomly with an average spacing $s$, and are identical without internal lossless so the $N$ scattering coefficients are all the same

$$
g_{n}=g_{1}=\left(\frac{\sigma_{s}}{4 \pi}-\frac{k_{0}^{2} \sigma_{s}^{2}}{16 \pi^{2}}\right)^{1 / 2}-i \frac{k_{0} \sigma_{s}}{4 \pi},
$$

where $\sigma_{s}$ is the scattering cross section. In the current investigations, $\sigma_{s}$ is considered an independent parameter within the constraint imposed by conservation of acoustic energy: $\sigma_{s} \leq 4 \pi / k_{0}^{2}=\lambda^{2} / \pi$. For the current calculations, the incident field $\psi_{0}(\mathbf{r})$ is a plane wave with amplitude $A$ and wave number vector $\vec{k}_{0}$, and the backscatter direction $(\phi=\varphi=0)$ is defined with respect to $\vec{k}_{0}$ as shown in Figure 1 . Three dimensional rectangular aggregations of scatterers with depth $X$, width $Y$, and height $Z$ have been the primary geometry considered this fiscal year because this geometry readily allows both nearfield and far-field investigations with a linear receiving array of length $L$ when the number of scatterers is limited to a few thousand.

To search for the presence or absence of ACBE, the scattered field $\psi_{s}(\mathbf{r})$ predicted by (1) is calculated at the elements of the receiving array, and aggregation-array distance $R$ is varied to put the receiving array in the near-field, where the array's beam-steering angle $\phi$ is relevant, or in the far-field of the aggregation, where the azimuthal scattering angle $\varphi$ is relevant. For near-field calculations, the results are presented as $B(\phi) /[B]_{\text {excluding peak }}$ vs. $\phi$, where $B(\phi)$ is the beamformed output of the receiving array, and $[B]_{\text {excluding peak }}$ is the array's average beamformed output in directions near backscatter but excluding the ACBE peak. The independent parameters of these investigations are $A, k_{0}, \sigma_{s}, s, R, L, \phi$ or $\varphi, X, Y$, and $Z$, but the current problem can be stated in dimensionless terms by normalizing field values with the incident wave amplitude $A$, and by using wave number-scaled lengths. This reduces the number of independent parameters on which ACBE may depend from ten to eight.

The current focus of this research effort is on determining and understanding the parametric dependence of the ACBE peak's height and width in the near field on the eight dimensionless parameters $\left(k_{0} \sigma_{s}^{1 / 2}, k_{0} s, k_{0} R, k_{0} L, \phi\right.$ or $\varphi, k_{0} X, k_{0} Y$, and $\left.k_{0} Z\right)$. In particular, the current simulations show the ACBE peak width is set by the dimensionless length $k_{0} L$ of the receiving array, while the peak enhancement depends on a combination of $k_{0} \sigma_{s}^{1 / 2}, k_{0} S$, and the beam steering angle between the center of the receiving array and the edge of the rectangular aggregation, $\phi_{\text {edge }}\left[=\tan ^{-1}(Y / 2 R)\right]$. 
These ACBE investigations are the current doctoral research of Ms. Adaleena Mookerjee. She is a US Citizen and a Ph.D. candidate.

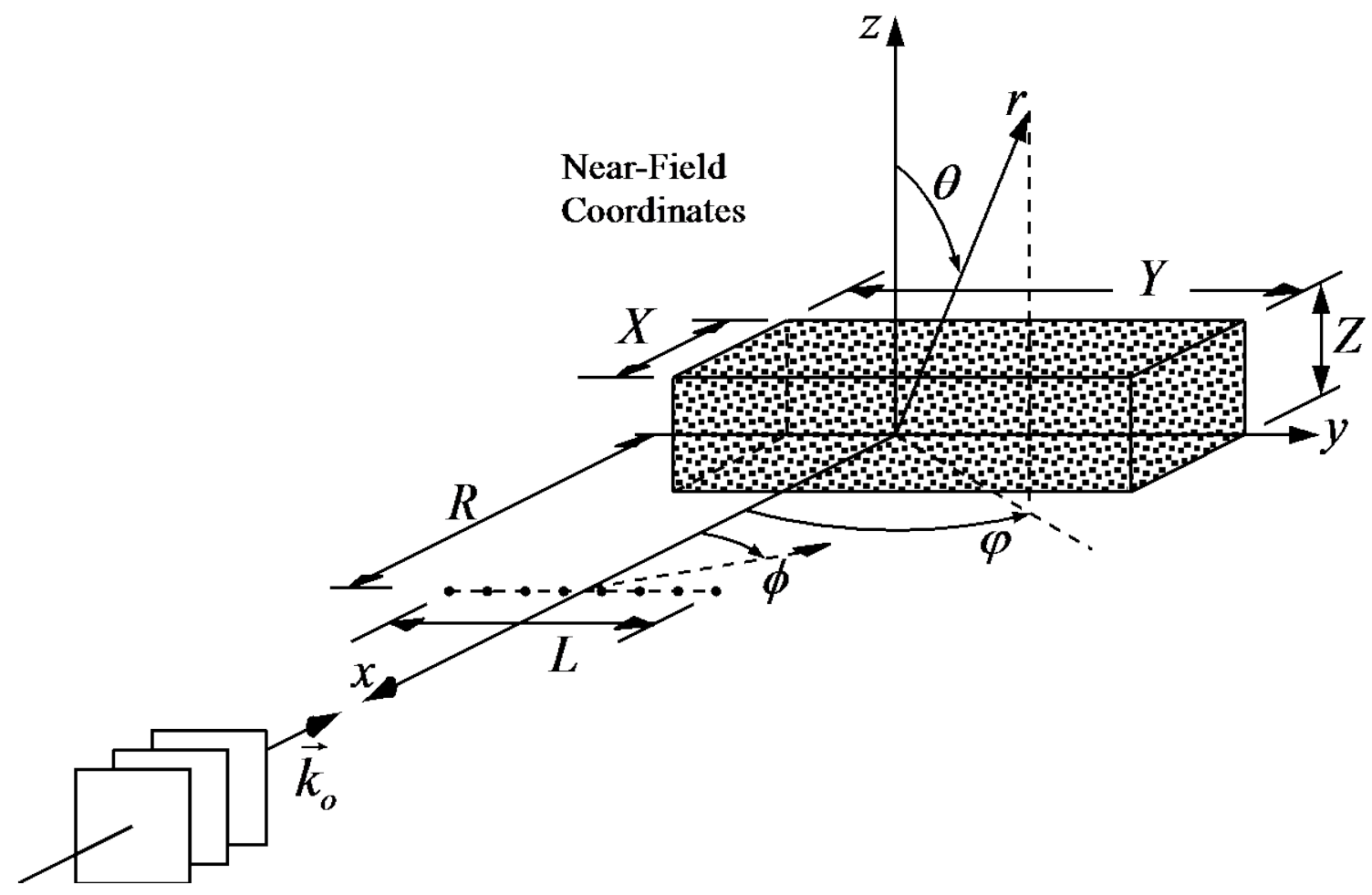

Figure 1. Geometrical configuration of the multiple scattering simulations. A plane wave with amplitude $A$ and wave number vector $\overrightarrow{\boldsymbol{k}}_{\boldsymbol{o}}$ impinges on a rectangular aggregation of scatterers with dimensions $X, Y$, and $Z$. Here $\varphi$ is the scattering angle defined from the origin of coordinates, and $\phi$ is the beam-steering angle defined from the broadside direction of the receiving array of length $L$ that lies a distance $R$ from the aggregation. The backscatter direction is $\varphi=\phi=0$.

\section{WORK COMPLETED}

In past years, the simulation code has been shown to properly recover Bragg scattering angles from uniformly spaced scatterers, and to conserve acoustic energy to better than $\pm 1 \%$ of the acoustic power incident on one scatterer even when thousands of scatterers are present. To complete the verification and validation of the simulations two more tests were undertaken: $(i)$ matching of the simulated effective wavenumber within the aggregation to Foldy's effective medium theory, and (ii) showing consistency of the simulated ACBE peak heights and widths with optics experiments. These tests were completed and an empirical coherence function for CBE was found along the way. In addition, the parametric dependence of the near-field CBE peak height on $k_{0} \sigma_{s}^{1 / 2}$ and $k_{0} s$ is now under investigation. The results are summarized in the next section and in a soon-to-be-submitted manuscript ${ }^{2}$.

\section{RESULTS}

Sample effective medium wave number matching results are shown in Figure 2. Here the real part of the ensemble-average acoustic pressure (black curve) inside a random aggregation of point scatterers is 
plotted along with the real part of the incident harmonic plane pressure wave (red curve) as a function of the incident-wave-number-scaled distance, $k_{0} x$, inside the aggregation. Here, the simulated wave number (wavelength) is slightly higher (lower), and this wave number change is predicted by Foldy's effective medium theory; $k_{e}{ }^{2}=k_{0}{ }^{2}+4 \pi N_{v}\left|g_{1}\right|$, where $k_{e}=2 \pi \lambda_{e}$ is the wave number inside the scattering aggregation, $\lambda_{e}$ is the wavelength inside the scattering aggregation, $N_{v}$ is the number of scatterers per unit volume, and $g_{1}$ is given by (4). For the conditions of these simulations, the ratio $k_{e} / k$ is predicted to be 1.0182, and the value for this ratio determined from the simulations, 1.0183 , is in good agreement with the value from theory.

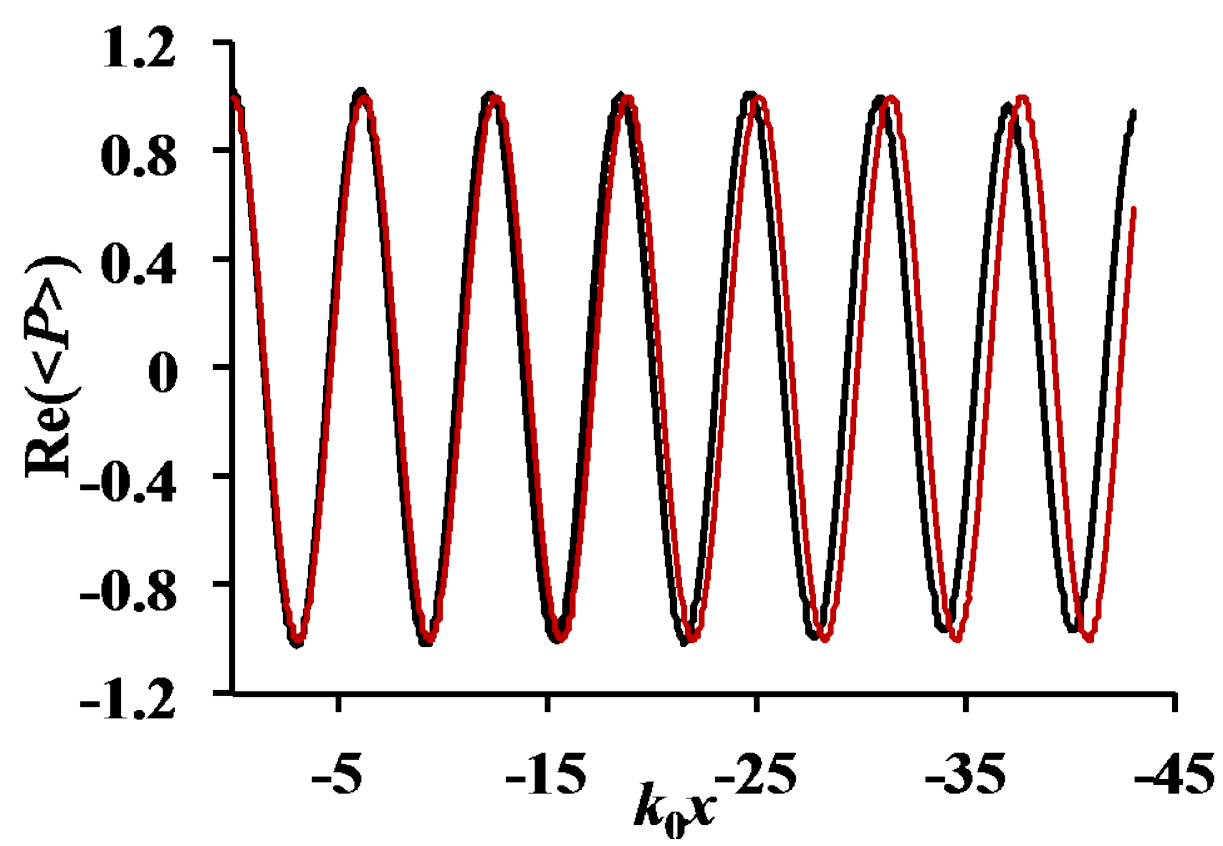

Figure 2. A comparison of the real part of the ensemble-average harmonic pressure (black curve) inside a random aggregation of point scatterers and the real part of the incident plane wave (red curve). The horizontal axis is the distance scaled by the wave number of the incident plane wave.

The wavelength inside the aggregation is slightly shorter, and simulation results shown here conform to the theoretically-expected wavelength change.

As a final validation test, results from the simulations were compared to optical CBE experiments ${ }^{3}$. This proved challenging for several reasons. The optics experiments involved polarized green laser light, trillions of $0.109-\mu \mathrm{m}$-diameter plastic beads, and an angular resolution of 0.25 milli-radians (mrd) while the simulations involved longitudinal acoustic waves, 3900 point scatterers, and an angular resolution of $\sim 2^{\circ}(30-40 \mathrm{mrd})$. To show that the results from the experiments and simulations were comparable, scattering cross sections $\left(k_{0} \sigma_{s}^{1 / 2}=0.23,0.21\right)$, average scatterer spacings $\left(k_{0} s=3.1\right.$, $3.2)$, and mean-free paths $\left(k_{0} l=925,926\right)$ were closely matched. To account for the difference in angular resolution, a single analytical coherence function was identified and used to determine the shape of the CBE peak using beamforming ${ }^{4}$ when the receiving array length is adjusted to alternately match the resolution of the optical experiments and the simulations. This coherence function is: 


$$
\Gamma\left(k_{0} \Delta y\right)=A_{d}^{2} \frac{\sin \left(k_{0} \Delta y\right)}{k_{0} \Delta y}+A_{b s}^{2}\left(1+\left(\frac{\Delta y}{\sigma_{y}}\right)^{2}\right)^{-1}
$$

where $\Delta y$ is the spatial shift variable, and $A_{d}, A_{b s}$, and $\sigma_{y}$ where chosen to produce the results shown in Figure 3. The green curves in both panels of Figure 3 come from the coherence function and both appropriately match the corresponding experimental and simulation results. Thus, the simulations and the experiments - although different in resolution - are consistent with each other.
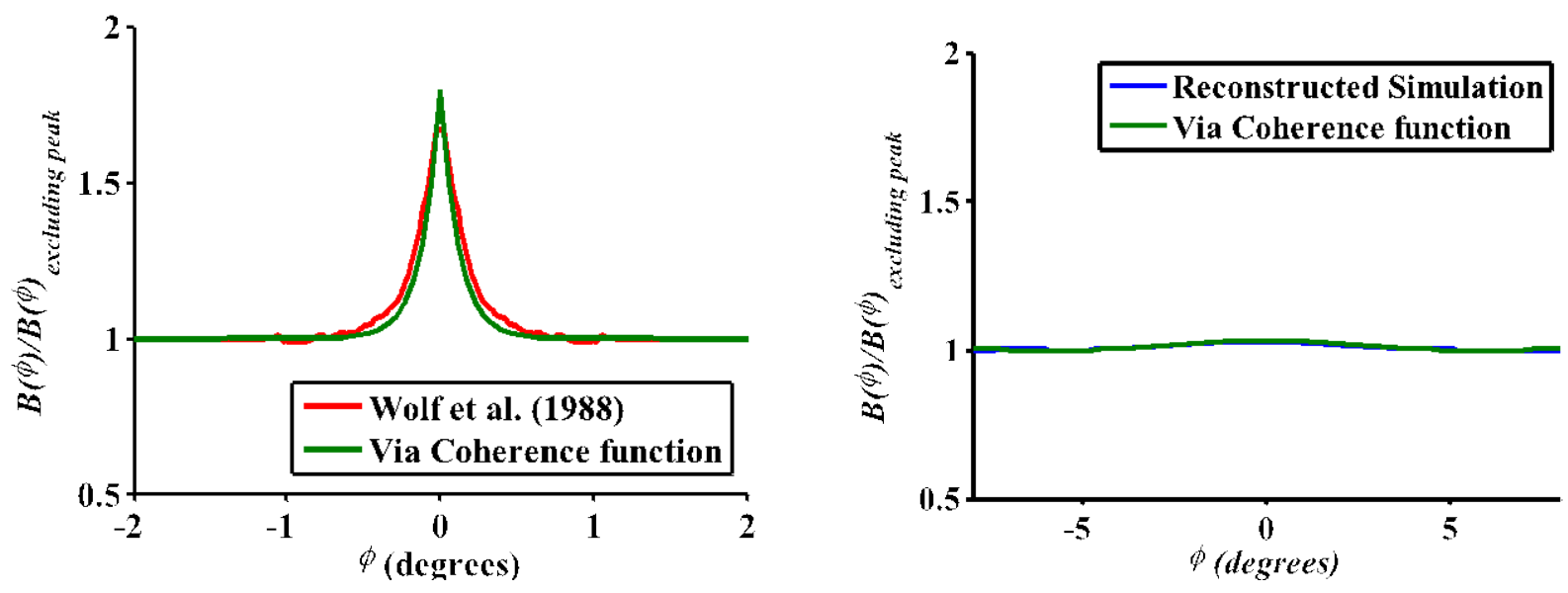

Figure 3. Ensemble averaged beamformed intensity ratio, $B(\phi) / B(\phi)_{\text {excluding peak, }}$, from the optics experiments (left) and from the simulations (right) versus angle $\phi$, where $\phi=0$ is the exact backscatter direction. The important difference between the two panels is the difference in the angular resolution of the receiving array $\left(0.25 \mathrm{mrd}\right.$ on the left and $2^{\circ}$ on the right). In both panels, the green curve is obtained from (5) using the same values of $A_{d}, A_{b s}$, and $\sigma_{y}$.Thus, the results are consistent even though the angular resolutions differ by approximately two orders of magnitude.

Overall, after four verification and validation checks, no deficiencies have been found in the simulations. However, while reaching the results shown in Figure 3, the reason for the prior findings of much larger than expected enhancements has been traced to the finite size and nominally flat front of the rectangular aggregation of scatterers used in the simulations and depicted in Figure 1. Current work involves production of a functional scaling for the peak ratio at $\phi=0$ in the near field of the aggregation based on simulation results for different combinations of $k_{0} \sigma_{s}^{1 / 2}$ and $k_{0} s$.

\section{IMPACT/APPLICATION}

In broad terms, this project ultimately seeks to determine if and how ACBE might be exploited for active sonar applications. In particular, if successful, it should prove useful for remote classification, because a large sonar return from a single large scatterer will likely not display ACBE while a similarly large sonar return from an aggregation of many small scatterers may display ACBE. Thus, 
this research effort may eventually impact how active sonar signals are processed and displayed for tactical decision-making related to classification.

\section{TRANSITIONS}

The results of this research effort should aid in the design of active sonar signal processors for tactical decision aids. However, at this time no direct transition links have been established with more applied research programs. Once the current simulation capability has been more fully exploited for parameter studies, and promising results have been obtained, a transition path through NRL or one of the Navy's Warfare Centers will be sought.

\section{RELATED PROJECTS}

This project is related to the other projects funded under ONR's 2010 basic research challenge program. In particular, the efforts by Prof. Feuillade in Chile and Prof. Sabra at Georgia Tech are most closely related.

\section{REFERENCES AND PUBLICATIONS}

[1] Foldy, L.L. (1945) "The multiple scattering of waves, I. General theory of isotropic scattering by randomly distributed scatterers," Physical Review Vol. 67, 107-119.

[2] Mookerjee, A. and Dowling, D.R. "Simulating acoustic coherent backscattering enhancement" to be submitted to J. Acoustic. Soc. Am., October 2014.

[3] Wolf, P.-E., \& Maret, G. (1985). Weak Localization and Coherent Backscattering of Photons in Disordered Media. Physical Review Letters Vol. 55, 2696-2699.

[4] Aubry, A., Derode, A., Roux, P., \& Tourin, A. (2007). Coherent backscattering and far-field beamforming in acoustics. Journal of the Acoustical Society of America Vol. 121, 70-77.

\section{HONORS AND AWARDS}

Ms. Mookerjee received a student presentation award for her talk on this topic at the 167th Meeting of the Acoustical Society of America in Providence, RI. 